\title{
Single-participant structural similarity matrices lead to greater accuracy in classification of participants than function in autism in MRI
}

\author{
Matthew J. Leming ${ }^{1,2^{*}} \mathbb{0}$, Simon Baron-Cohen ${ }^{1}$ and John Suckling ${ }^{1}$
}

\begin{abstract}
Background: Autism has previously been characterized by both structural and functional differences in brain connectivity. However, while the literature on single-subject derivations of functional connectivity is extensively developed, similar methods of structural connectivity or similarity derivation from T1 MRI are less studied.

Methods: We introduce a technique of deriving symmetric similarity matrices from regional histograms of grey matter volumes estimated from T1-weighted MRIs. We then validated the technique by inputting the similarity matrices into a convolutional neural network (CNN) to classify between participants with autism and age-, motion-, and intracranial-volume-matched controls from six different databases (29,288 total connectomes, mean age $=30.72$, range $0.42-78.00$, including 1555 subjects with autism). We compared this method to similar classifications of the same participants using fMRI connectivity matrices as well as univariate estimates of grey matter volumes. We further applied graph-theoretical metrics on output class activation maps to identify areas of the matrices that the CNN preferentially used to make the classification, focusing particularly on hubs.

Limitations: While this study used a large sample size, the majority of data was from a young age group; furthermore, to make a viable machine learning study, we treated autism, a highly heterogeneous condition, as a binary label. Thus, these results are not necessarily generalizable to all subtypes and age groups in autism.

Results: Our models gave AUROCs of 0.7298 (69.71\% accuracy) when classifying by only structural similarity, 0.6964 (67.72\% accuracy) when classifying by only functional connectivity, and 0.7037 (66.43\% accuracy) when classifying by univariate grey matter volumes. Combining structural similarity and functional connectivity gave an AUROC of 0.7354 (69.40\% accuracy). Analysis of classification performance across age revealed the greatest accuracy in adolescents, in which most data were present. Graph analysis of class activation maps revealed no distinguishable network patterns for functional inputs, but did reveal localized differences between groups in bilateral Heschl's gyrus and upper vermis for structural similarity.
\end{abstract}

Conclusion: This study provides a simple means of feature extraction for inputting large numbers of structural MRIs into machine learning models. Our methods revealed a unique emphasis of the deep learning model on the structure of the bilateral Heschl's gyrus when characterizing autism.

*Correspondence: mleming@mgh.harvard.edu

2 Present Address: Center for Systems Biology, Massachusetts General

Hospital, 149 13th Street, Boston, MA 02129, USA

Full list of author information is available at the end of the article permits use, sharing, adaptation, distribution and reproduction in any medium or format, as long as you give appropriate credit to the original author(s) and the source, provide a link to the Creative Commons licence, and indicate if changes were made. The images or other third party material in this article are included in the article's Creative Commons licence, unless indicated otherwise in a credit line to the material. If material is not included in the article's Creative Commons licence and your intended use is not permitted by statutory regulation or exceeds the permitted use, you will need to obtain permission directly from the copyright holder. To view a copy of this licence, visit http://creativecommons.org/licenses/by/4.0/. The Creative Commons Public Domain Dedication waiver (http://creativeco mmons.org/publicdomain/zero/1.0/) applies to the data made available in this article, unless otherwise stated in a credit line to the data. 
Keywords: Autism, Deep learning, Functional connectivity, Structural similarity

\section{Background}

Voxel-based morphometry (VBM) [1] is a means of detecting structural differences in brain anatomy from T1-weighted MRI across groups. In VBM, images are registered to the same coordinate space and segmented into grey matter, white matter, and cerebrospinal fluid (CSF) volumes, before comparisons are made across voxels or groups of voxels using standard statistical tests. Due to its robustness and effectiveness, VBM has enjoyed significant popularity since it was first introduced $[2,3]$. Structural covariance networks [4] correlate tissue volumes estimated by VBM in regions across groups of participants to describe relationships that are interpreted as measures of structural integrity or developmental coherence of the brain. These networks have been coupled with gene expressions [5] and correlated with diseaserelated alterations in brain topology [6], but their underlying neurophysiology is still an active area of study.

While there have been several cross-sectional findings of structural brain differences in autism [7-9], these have not been substantiated by a larger-scale analysis [10]. Indeed, characterizations of brain structure in autism have been inconsistent across studies of small sample sizes, although differences at different ages may explain some of this variation [11]; for instance, increased amygdala volumes have been reported in children with autism $[12,13]$, but not adults [8]. A meta-analysis of VBM studies in autism found disturbance of brain structure in the lateral occipital lobe, the pericentral region, the right medial temporal lobe, the basal ganglia, and proximate to the right parietal operculum [9]. Small-scale studies in children with autism have found altered structural covariance in areas associated with sensory, language, and social development. Altered structural covariance has been found between sensory networks, the cerebellum, and the amygdala in autism [14]. In children, [15] found that structural covariance indicated localized reductions within fronto-striatal and parietal networks and decreases in ventral and superior temporal grey matter, suggesting abnormalities in the anatomy and connectivity of limbic-striatal (i.e. social) brain system. Language ability correlated with cortical structure and covariance [16], and associations with language development are further supported by studies showing abnormal development of the Heschl's gyrus [17], an area where functional activation has been associated with development of "inner speech" [18]. In adults with autism, structural covariance has shown decreased centrality in cortical volume networks [19].
Autism has been consistently associated with differences in brain function [20,21]. Efforts to find differences in functional connectivity relative to neurotypical control groups have characterized autism as exhibiting underconnectivity, and thus greater segregation of functional areas [22-27]. Other studies, mostly of children and adolescents, found evidence of over-connectivity in specific areas of the brains of those with autism [28-33], locating hyperconnectivity to the posterior right temporo-parietal junction [29] and in striatal areas and the pons [30,31]. [34] posited that autism is likely characterized by a mix of hyper- and hypo-connectivity traits.

\section{Machine learning}

Machine learning has found multiple applications to the analysis of brain images in recent years, including pre-processing, segmentation, and diagnostics. Of great interest has been whole-brain phenotypic classification, in which MRI data of two or more phenotypes (such as sexes, or a diseased group and healthy controls) are trained and classified with a machine learning algorithm. Such studies most often include four steps: (1) selection of MRI modality and derived features that are sensitive to the problem at hand; (2) feature extraction, to reduce data dimensionality; (3) inputting features to train a machine learning model with the selected architecture; and (4) classification and interpretation.

MRI feature extraction is most often performed using techniques previously developed in image analysis, and the specific method is dependent on the selected modality and features. For instance, based on a large body of research and predictable dimensionality reduction [35, 36], it is common to use for classification functional connectivity matrices [37-39] representing correlations in time-series between pre-defined regions derived from blood oxygenation level-dependent (BOLD) sensitive fMRI. Likewise, to classify diffusion weighted images (DWI) it is common to use structural similarity matrices representing measures of white matter tracts traversing the brain between specific regions [40-42].

However, while there exists several consensus methods for deriving connectivities from fMRI $[36,43]$ and DWI [35] (though this is still an active area of research $[44,45])$, analogous means of connectivity-based dimensionality reduction for T1-weighted structural MRI [46, 47] are not as widely used, even though this is the most common [48] modality available to study. One reason for the lack of common methodology is that reductions from three-dimensional data to network representations 
with meaningful physiological interpretation are more difficult to produce than reductions of four-dimensional data. In most existing feature extraction methods for T1-weighted MRI, extracted features are typically independent, univariate measures from regions of interest, such as cortical thickness and surface curvature. However, the lack of a connectivity metric leads not only to the loss of spatial encoding seen in network representations, but fewer features overall (i.e. for $N$ ROIs, connectivities output $O\left(N^{2}\right)$ features while univariate measurements output $O(N)$ ), reducing effectiveness for machine learning.

For this study, we designed a similarity metric that reduced T1-weighted MRIs to a network representation without an a priori physiological interpretation, then applied it a dataset of autistic individuals and neurotypical controls. We applied this method to an extremely large dataset of participants with autism, representing a disorder for which structural characterization had proven difficult [49-52].

\section{Machine learning in autism}

Previous machine learning studies of autism have achieved classification accuracies that widely varied depending on the modality used, sample size, data quality, selected methods, and diagnostic criteria. A recent study [53] of 106 high-risk infants between 6-12 months linked brain volume overgrowth to the emergence and severity of autism symptoms, using a deep learning algorithm capable of predicting autism with $81 \%$ specificity and $88 \%$ sensitivity using brain surface information. Another study by the same group [54] found that autism could be predicted in 59 6-month-old infants with $81.8 \%$ sensitivity using functional imaging. In the general population, efforts in single-participant classification of autism from MRI data have had mixed results [49, 55-60], with studies rarely exceeding $80 \%$ classification accuracy [34]. Again, however, this varies substantially by modality and which site data were collected [50]. In a recent study, Eill et al [61] performed a classification on individuals with autism and neurotypical controls using structural MRI, DWI, and fMRI data, finding that features derived from fMRI provided the highest accuracies with a support vector machine. They did, however, encounter the issue of fMRI feature extraction simply producing more variables than its structural counterparts, offering the machine learning model more information to work with, although attempts were made to mitigate this issue.

\section{Experiments}

In the present study, we present a simple method of deriving structural similarity matrices from T1-weighted MRI. Our method compared the distributions of grey matter in pairs of parcellated areas of T1-weighted MRI. This metric acts as an indirect means of encoding relative size of grey matter volume while also being an effective means of dimensionality reduction that allows for T1-weighted MRIs to be encoded into a machine learning model. We describe our dataset, including acquisition and pre-processing methods. We validated these data using a machine learning model previously used in Leming and Suckling 2020 [62] and made comparisons to the classification accuracy using the corresponding fMRI connectivity matrices of the same participants, as well as lower-dimensional data consisting of grey matter volumes averaged within regions. Finally, we used the output class activation maps (CAMs), combined with graph theoretical techniques, to understand which parts of the brain the model focused on, and whether simple linear regression models could spot the same qualities in these data. We further describe a means of combining our structural similarity matrices with functional connectivity matrices in the same machine learning model to yield improved accuracy. Additionally, we show how the autism classification performance differed across age groups.

\section{Methods \\ Dataset}

We used a dataset comprised of 29,288 total instances each with a structural MRI and a functional MRI in both task-activated and task-absent (rest) conditions. (Note that instances were acquired from the same participant.) In total, 1555 data points were from participants with autism. These data were drawn from six different databases: Open fMRI, the UK BioBank, ABIDE I, ABIDE II, NDAR (minus ABCD), and ABCD (Table 1). Autism labels were collected in different ways depending on the format of the respective databases; for instance, ABIDE and BioBank had autism data already labelled, while NDAR and ABCD required keyword searches across different studies contributed by different groups; in such cases, unclear labels resulted in exclusion of data. Covariates of age, sex, and task were also compiled.

\section{Pre-processing and functional feature extraction}

Functional data were pre-processed using SpeedyPP [63]. Data were first skull stripped using the Analysis of Functional Neuroimages (AFNI) toolbox. Motion was regressed from time series using wavelet despiking [63]. Data were then registered to the stereotaxic space of the Montreal Neurological Institute (MNI), after which they were overlaid on the 116-area AAL parcellation. Functional datasets with greater than $10 \%$ 
Table 1 Statistics for each dataset used

\begin{tabular}{|c|c|c|c|c|c|c|c|c|c|c|c|}
\hline \multirow[b]{2}{*}{ Collection } & \multirow[b]{2}{*}{ Subj. } & \multirow[b]{2}{*}{ Conn. } & \multirow[b]{2}{*}{ Rest } & \multirow[b]{2}{*}{ Task } & \multicolumn{2}{|l|}{ Age } & \multirow{2}{*}{ Mean } & \multirow[b]{2}{*}{ Stdev } & \multicolumn{2}{|l|}{ Sex } & \multirow[b]{2}{*}{ Autism } \\
\hline & & & & & Min & Max & & & $\mathbf{F}$ & $\mathbf{M}$ & \\
\hline$A B C D$ & 1049 & 5142 & 2296 & 2846 & 0.42 & 11.08 & 10.12 & 0.69 & 2474 & 2668 & 61 \\
\hline ABIDE & 412 & 412 & 412 & 0 & 6.00 & 45.00 & 17.00 & 7.16 & 45 & 367 & 181 \\
\hline ABIDE II & 682 & 717 & 717 & 0 & 5.22 & 55.00 & 14.39 & 7.39 & 169 & 548 & 350 \\
\hline BioBank & 9791 & 9791 & 9791 & 0 & 40.00 & 70.00 & 55.00 & 7.51 & 5178 & 4613 & 4 \\
\hline NDAR & 1050 & 7958 & 5531 & 2427 & 0.58 & 55.83 & 18.71 & 7.80 & 3816 & 4142 & 930 \\
\hline Open fMRI & 1194 & 5268 & 820 & 4448 & 5.89 & 78.00 & 27.12 & 10.24 & 2346 & 2479 & 29 \\
\hline Total & 14178 & 29288 & 19567 & 9721 & 0.42 & 78.00 & 30.72 & - & 14028 & 14817 & 1555 \\
\hline
\end{tabular}

regional dropout, or either structural or functional datasets that otherwise failed the pre-processing stage, were excluded. The remaining datasets are presented in Table $1.116 \times 116$ functional connectivity matrices were estimated using Pearson correlation on the averaged timeseries within a region.

\section{GM volume and single-participant structural similarity matrices}

To estimate grey matter volume distributions in each area in the AAL parcellation, structural MRI were first skull stripped using tools from AFNI, then registered to MNI space. Grey matter volumes were estimated using FSL VBM. We measured the similarity, $s$ between two regions by comparing the distributions of nonzero voxel values within each AAL region $(u$ and $v)$, using the following equation:

$$
s=\inf _{\pi \in \Gamma(u, v)} \int_{x, y \in \mathbb{R} \times \mathbb{R}}|x-y| d \pi(x, y)
$$

in which $\Gamma(u, v)$ is the set of distributions on $\mathbb{R} \times \mathbb{R}$ whose marginals are $u$ and $v$ [64]. This is simply the Wasserstein metric, which indicates the minimal amount of work necessary to transport one distribution to another. (In describing this metric, the two different distributions are often described as piles of dirt-hence its alternative name, "Earth-Mover's distance".) Prior inspection of these areas showed that the distribution of GM volume values followed no clear statistical distribution, and so this is an ideal metric as it nonparametically compares two statistical distributions, regardless of relative region sizes. A similar metric, the Kullback-Leibler divergence, has previously been used in brain morphology comparisons [46, 47], but this metric requires the estimation of a probability density function approximating grey matter distributions, because it is sensitive to histogram binning, whereas the Wasserstein distance is less so [65] and can be applied directly on discrete data. While this similarity metric does do away with spatial encoding and thus eliminates crucial information such as curvature, it acts as a comparison of the distributions of grey matter volumes between two areas in an easily understood way, and at a low computational cost. An illustration of this is shown in Fig. 1. Finally, univariate grey matter volumes were estimated by averaging nonzero voxels of GM volumes within each AAL region (effectively, the average of the distributions compared to find the structural

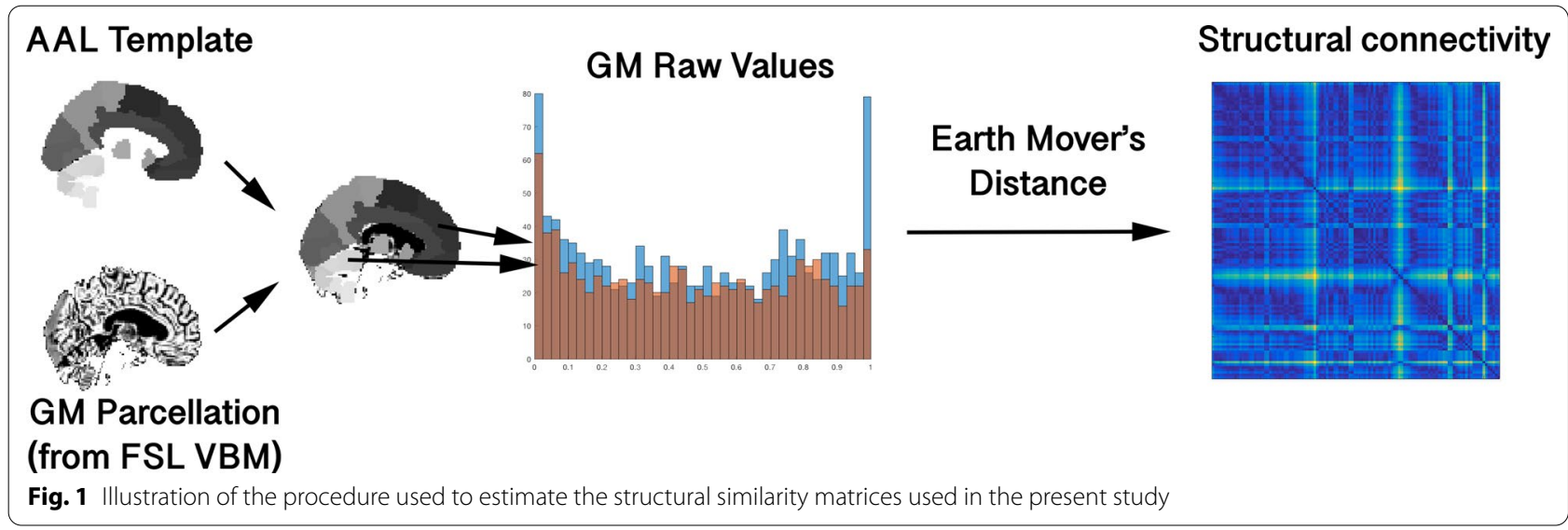


similarity metric), producing a $116 \times 1$ array of numbers. Because this needed to match the dimensionalities of the structural similarity and functional connectivity to be input into the $\mathrm{CNN}$, these values were repeated to form a $116 \times 116$ matrix.

\section{Comparison of structural similarity with functional connectivity}

To determine whether functional connectivity and our novel structural similarity metric shared information in common, we correlated functional matrices from each instance with their corresponding structural matrices, in 10,000 random function/structural pairings (i.e. which were derived from the same subject) from across the whole dataset. We then compared these correlations with a null model estimated by correlating random pairings of functional and structural matrices (i.e. which were derived from different subjects). This was done by comparing the two sets of $10,000 \mathrm{R}$ values with a t-test, indicating the amount of common information encoded by both structural similarity and functional connectivity.

\section{Machine learning model and training}

We classified autistic individuals and neurotypical controls using, separately, structural similarity, grey matter volume, and functional connectivity measurements, as well as a model that combined structural and functional connectivities. We employed the model and training scheme described in Leming and Suckling 2020 [62] (submitted). This used an ensemble of 300 convolutional neural networks that each scrambled each symmetric $116 \times 116$ input matrix into a randomly ordered $115 \times 58$ rectangle, losing some spatial encoding information while avoiding biases in output class activation maps and maintaining the regularization effect of convolutions. Additionally, the use of CNNs allowed for the encoding of multiple connectivities (in this case, structural and functional) in different input channels, taking advantage of data structures that can alternatively encode RGB images. Matrices were then input to a CNN with 256 filters of shape $1 \times 58 \times 1$. This convolved $58 \times 3$ random values of the matrix which was then fed into three dense layers, each with 64 hidden units, with batch normalization layers, rectified linear unit (ReLU), and 0.5 dropout between them. Finally, the data was binary classified through a softmax layer.

In building training, test, and validation sets for our models, a multivariate class balancing scheme was used [62]. For each autistic instance sampled in a given set, we sampled a neurotypical control participant of the same sex and data collection and with a statistically similar fMRI mean framewise displacement, intracranial volume, and age. The class balancing scheme further divided data into test, training, and validation sets for each model in the ensemble, ensuring participants with multiple functional connectomes were allocated to the same set. Each model was trained on an Adam optimizer for 100 epochs, with a copy of the model being saved at each epoch; the model that yielded the highest accuracy on the validation set was selected, and the prediction on the test set was the final accuracy reported.

For each classification task, we trained 300 independent CNNs, each with their own independently sampled and balanced training/test/validation set, then averaged their predictions for each data instance that fell within at least one test set. This effectively created an ensemble of CNNs, with each data instance having at least one $\mathrm{CNN}$ making a prediction. The final AUROC was derived by averaging predictions for each data instance that fell within a test set. Cross-contamination between training and test sets was prevented because the only predictions sampled were those of the independent model and its associated test set. Different aggregated AUROCs were recorded when the specific number of models in the ensemble varied; when adding models to the ensemble, the AUROC from the aggregated models increased in a predictable way. The AUROCs from between 20 and 300 models were fit to a logarithmic curve with a hard limit in order to predict the projected highest AUROC possible in the limit of a large number of models.

As a result of forced class balancing, each model in the ensemble used an independent subset of approximately 1600 instances, which was split between training, test, and validation sets at a ratio of 4:1:1. Because the multivariate class balancing scheme has stochastic elements and was run independently for each of the 300 models, there were small variations in the sizes of the final sets for each model, though variations were small (with a maximum size difference of about 50) and the size of the training set was overall consistent. For each model, different splits were used between training, test, and validation sets. As an effect of this balancing scheme, data from Open fMRI and the UK BioBank, having few participants overall with a diagnosis of autism, were included only infrequently, while data from ABIDE I and II, ABCD, and NDAR were frequently represented.

In total, four cross-sectional classification tasks were undertaken (Table 2), specifically: with structural similarity; with grey matter volumes; with functional encoding; and by combining structural and functional connectivities.

\section{Class activation map analysis}

Using the Guided Gradient Class Activation Map (GradCAM) algorithm [66], which displays areas of the input data most salient in classification, we measured the class 

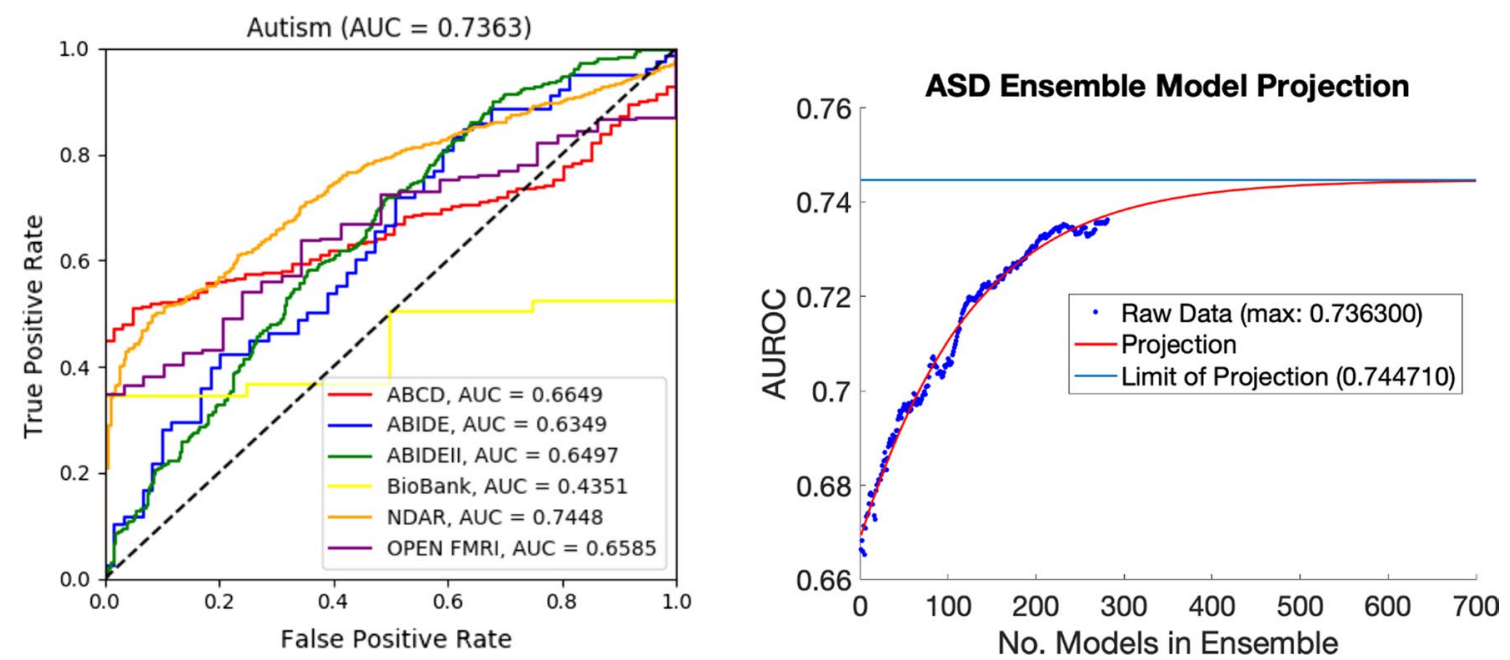

Fig. 2 Left: overall AUROCs of each dataset included in the analysis for the structure/function ensemble. Right: projection of the limit of the structure/function/age ensemble models, given data for ensembles of one to 300 models. Adding independent models ad infinitum would result in a maximum predicted $A U R O C$ of 0.745

activation of each data point in each model proposed, and then averaged these maps generating a $116 \times 116 \mathrm{CAM}$ for both structural and functional connectivity, as well as a $116 \times 1$ map for grey matter volume. We correlated the structural and functional CAMs to the measured effect size of differences between autism and neurotypical controls for our connectivity data, as a way to determine the similarity of CAMs to conventional statistics.

Next, we isolated hubs in the $116 \times 116$ CAMs. To do so, we first measured the edge betweenness centrality of each edge in our CAMs. We then grouped these values into different communities by maximizing modularity of the edge betweenness values (Brain Networks Toolbox [67]). This procedure identified which hubs were most focused on by the classifier.

\section{Results}

\section{Training}

Accuracies and AUROCs on the test set are given in Table 2.

Table 2 Respective AUROCs and accuracies of ensemble models on different combinations of data

\begin{tabular}{lll}
\hline Modality & AUROC & Accuracy \\
\hline Structural conn., function & 0.7354 & 69.3980 \\
Structural conn. & 0.7298 & 69.7062 \\
Function & 0.6964 & 67.7180 \\
Structure (GM vols) & 0.7037 & 66.4228 \\
\hline
\end{tabular}

Classification resulted in a higher AUROC for structural than functional connectivities: 0.7298 and 0.6964, respectively. Classification on univariate grey matter volumes resulted in an AUROC of 0.7037, outperforming functional classification while underperforming structural similarity classification, although this might be expected considering its lower dimensionality. Combining structure and function resulted in an AUROC of 0.7354 (Fig. 2, left), with a projected upper limit of the AUROC of 0.745 (Fig. 2, right).

Figure 3 shows the classification results across different age groups, reflecting the large disparities in age ranges present in the accumulated dataset, as well as the heightened model performance for those age ranges for which the most data was present. This reflects both the disparities in autism characterization across development as well as the likelihood of increased accuracy with more heterogeneous datasets.

\section{Class activation map analysis}

When comparing the output CAMs to their respective functional and structural effect sizes, no statistically significant correlation was observed, and thus, the machine learning model relied very little, if at all, on differences detectable by conventional statistics (Fig. 4).

CAMs for structural and functional connectivities, sorted by different detected communities after edge betweenness centrality was measured, are shown in Figs. 5 and 6. Structural CAMs showed five distinct groupings, each with distinct hubs that each centred on 


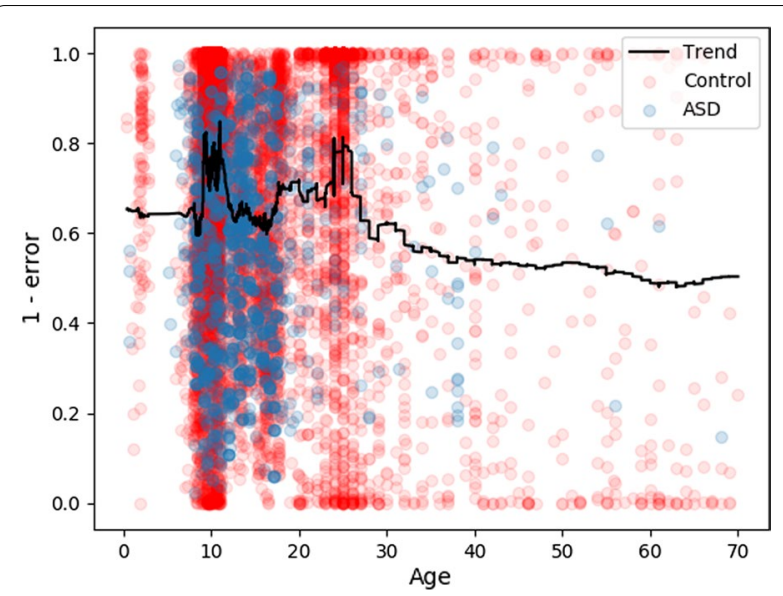

Fig. 3 Relative classification error in the structure/function/age ensemble model, plotted against age. Each point in the graph represents the averaged classification error of the datapoint across each model in which it was included in the test set. Thus, more controls are represented which were each used individually in fewer models, while the autism datasets are fewer but were generally used in more models. This represents that accuracy was generally higher in the developmental age groups, likely because more data was present for those groups

one or two localized areas, including the left and right Heschl's gyrus, the upper vermis, the right frontal-medial orbital gyrus, the right pallidum, and the left putamen. The strongest activations were found in left Heshcl's gyrus.

Localization was also found, though less distinctly, in functional hubs, notably the left inferior parietal lobe, the left middle temporal lobe, the left olfactory bulb, and the upper vermis. However, focus on particular hubs was not a distinctive feature.

CAMs for grey matter volumes are shown in Fig. 7. These results had very little in common with the structural similarity results, with the strongest five activated areas in the right supplementary motor area, the right middle frontal lobe, the right precentral sulcus, the left insula, and the inferior frontal gyrus triangularis.

\section{Discussion}

This study proposed a new feature extraction method for inputting structural MRIs into a network-based machine learning model, as well as applicable analysis methods to detect areas of that were particularly involved in determining the classification. Estimating single-participant structural similarity matrices from T1-weighted images without supplementary modalities such as DWI or fMRI is uncommon, and research in this area is ongoing [46, $47,68]$. In structural covariance, VBM data are used to produce inter-regional relationships at a group level, but this is inapplicable at a single-participant level, which is necessary to make structural MRIs applicable to machine learning models. The proposed method provides a means of doing so.

In developing this method, other means of estimating single-participant connectivity matrices from T1-weighted MRI were considered, such as estimating the correlation between different univariate measurements (cortical thickness, curvatures, and so on) of the structural image $[44,45]$, but this was too computationally intensive for a large dataset. Another method was investigated that involved finding the difference between group structural covariance matrices with and without a certain participant. While classifications on these matrices were successful, the matrices themselves varied to such an extent that the output CAMs were inconsistent; rather than describing physiological features, these matrices described deviations of individuals' physiological features, and as a result the matrices themselves had no consistent features that could be locally focused on by our DL model. The use of the Kullback-Leibler metric used in Kong et al [46] was also considered, but for the reasons described above, the Wasserstein metric was decided upon because of its simplicity and effectiveness in classification.

In the univariate grey matter volume results, the CAMs highlighted the right supplementary motor

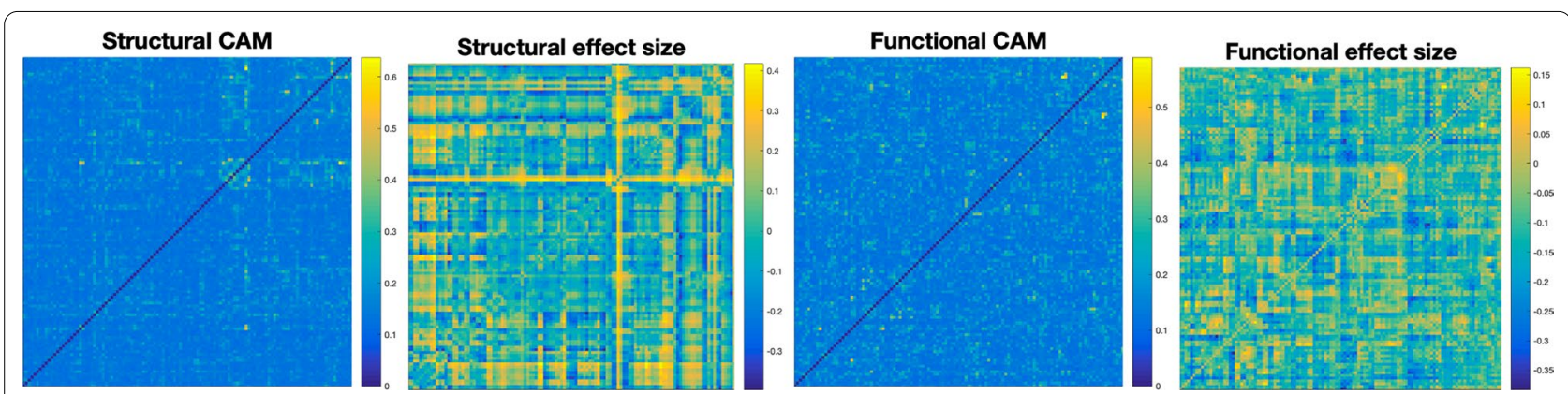

Fig. 4 Comparison of the effect size of differences between raw matrix values between groups and the averaged class activation maps. Most of the edge differences passed a nonparametric statistical significance test. When comparing the CAM matrix and the effect size matrices using either linear or nonparametric correlation, neither had any statistically significant associations with one another 


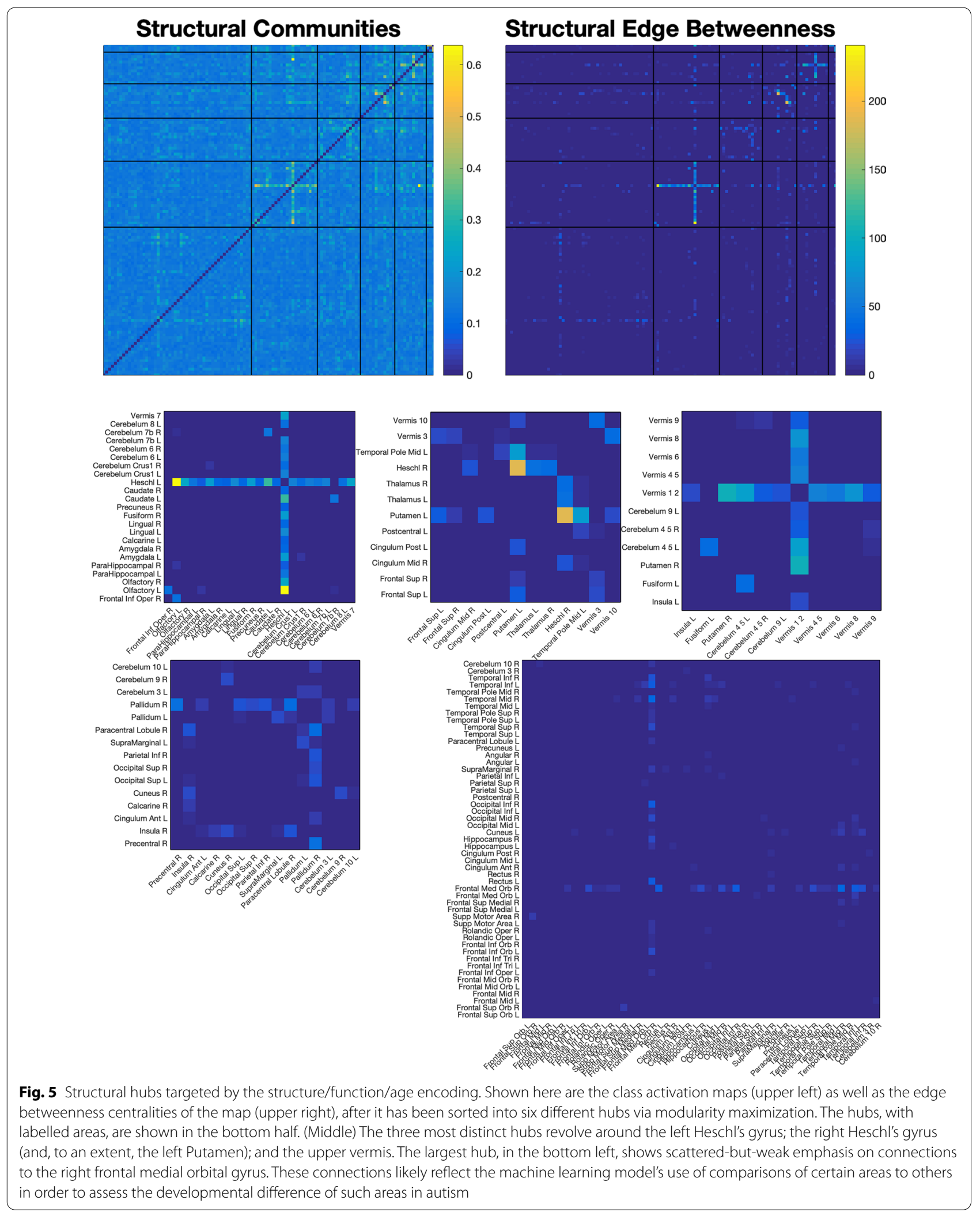




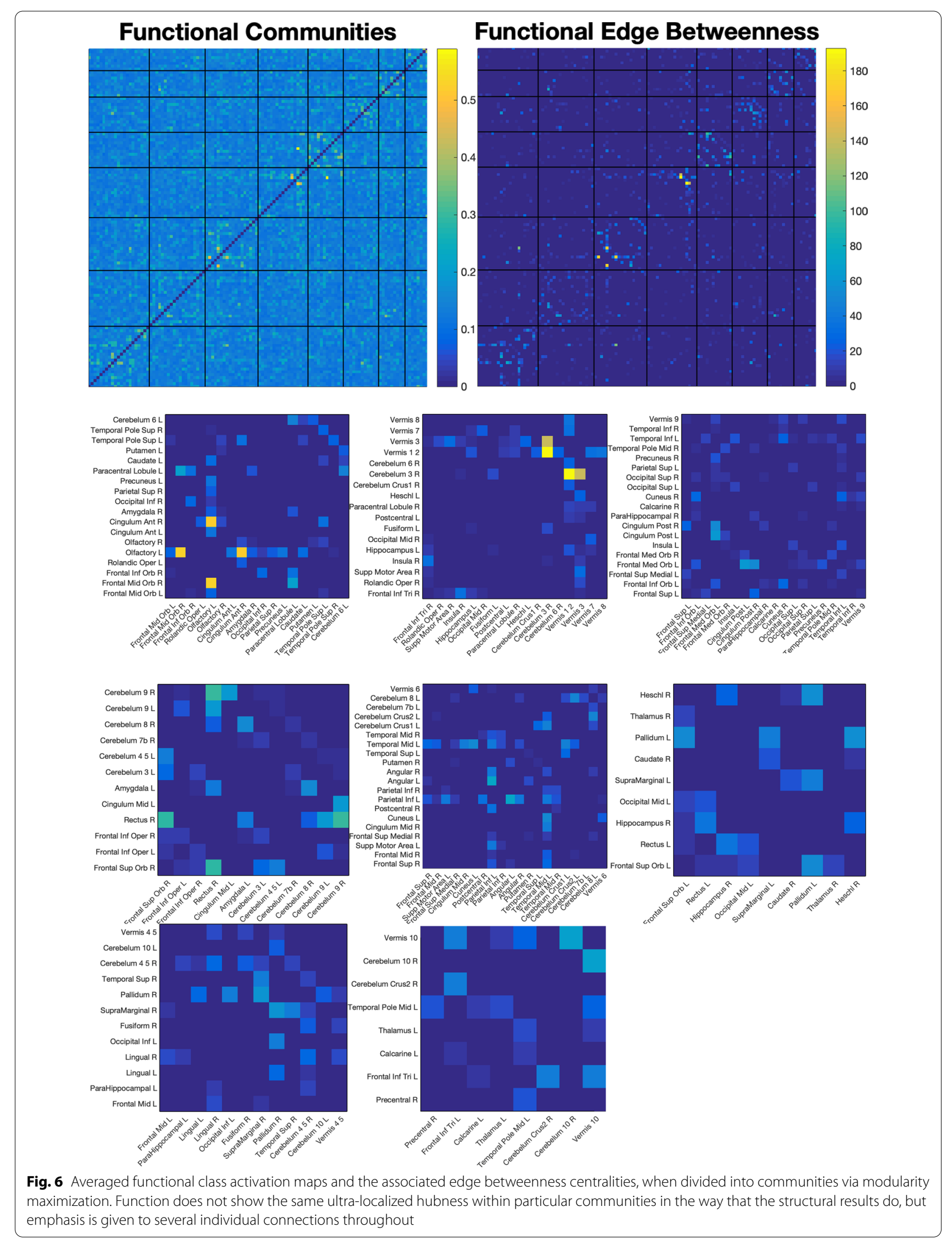




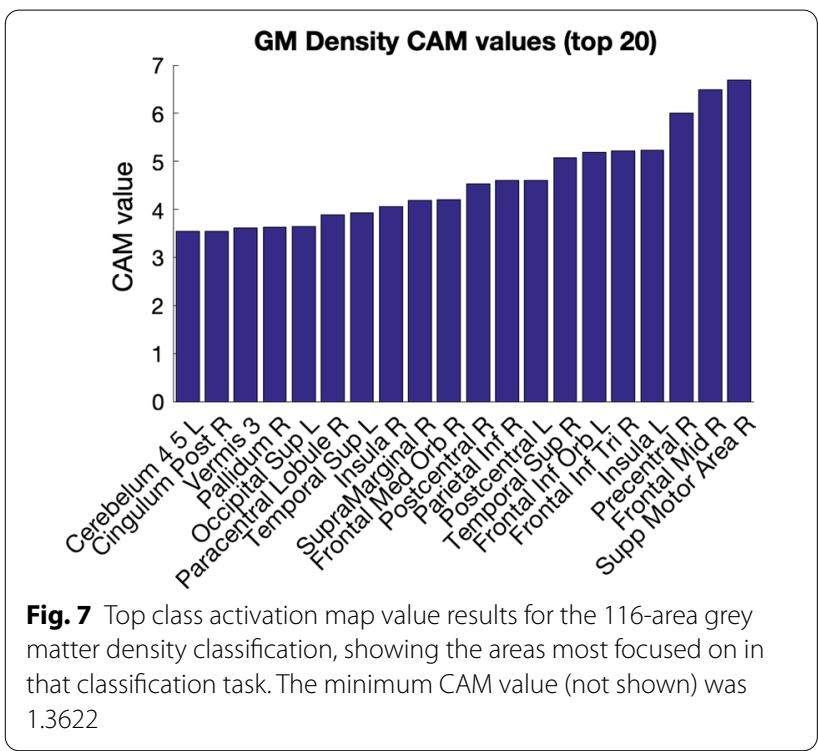

area, right mid frontal lobe, right precentral sulcus, left insula, right frontal inferior triangularis, left frontal inferior orbital lobe, and the right superior temporal lobe. (The top 20 areas are shown in Fig. 7.) Comparing the CAM emphasis of the grey matter volumes to the meta-analysis of autism VBM studies in [9], which found six areas with consistently altered grey matter volumes, some similarities can be seen, notably in the right superior temporal lobe where grey and white matter volume differences in the right medial temporal lobe and the left post central gyrus.

Functional analysis did not reveal a pattern of local hubness characterizing structural connectivity differences, but rather focused on specific connections. However, a number of general functional communities were identified (Fig. 6). Meta-analyses of studies in functional connectivity differences associated with autism have not found consistent differences in the brain, but rather in network-wide measures [34]. The lack of hub emphasis in functional results may be additional evidence of network-wide, rather than localized differences between autism and neurotypical control groups seen in other recent findings [69].

In structural similarity, three definitive hubs were identified: left Heschl's gyrus, right Heschl's gyrus, and the upper vermis. The right pallidum and fronto-medial orbital region also showed relatively strong local hubs, though to a lesser degree. Emphasis of the Heschl's gyrus is in agreement with recent studies in developmental autism, having been implicated previously as an area that develops atypically in autistic children [17]. Function of the area has been associated with development of "inner speech" [18], indicating a difference in development of language capabilities. Our findings differ in that they found this emphasis in structure and not function, but this may be reflective of the lower variability of differences across a single area in the development of grey matter as opposed to function, which likely varies far more across participants, and age groups. The cerebellum, meanwhile, has consistently been cited as an area of difference between individuals with autism and neurotypical controls during development [11], as well as an area of difference in structural covariance associated with autism [14].

The structural similarity CAMs resulting from this study revealed an emphasis on a number of distinct and localized areas, and these areas were clarified by use of an edge centrality measurement combined with modularity maximization to isolate hubs. The edge betweenness step was added by necessity to place extreme emphasis on a smaller number of more central edges, and only then could modularity maximization isolate hubs in a meaningful way (see Fig. 5).

The structural similarity method's efficacy with the machine learning model suggests that it encoded practically useful information about brain structure, but the interpretation of what these structural hubs indicate physiologically is more complicated. While some correlation is present (Fig. 8) the functional connectivity and structural similarities show largely different patterns. Furthermore, considering that the method used to estimate structural similarity was a similarity metric, the emphasis on these hubs was less likely an indication that they were centres of a physiological brain network characterizing autism. Because the strength of connections was a comparison of grey matter distributions, it is more likely that connections to the identified hubs were used by the machine learning algorithm as a proxy for detecting subtle changes in the morphology of grey matter within those specific regions. Edges connecting to these structural hubs were probably an indirect indication of differences in grey matter between two areas, and the individual connections themselves would not indicate any special physiological relationship, but rather a comparison of grey matter volume. However, this still means that the hubs themselves were important in characterizing autism, and, as this work has shown, it has clear utility in the context of machine learning. This structural similarity metric may simply be viewed as a way of encoding relative spatial information about the morphology of individual areas of the brain.

The univariate grey matter volume results further complicate interpretation because areas different from the structural similarity results were emphasized by the CAMs, even though both univariate grey matter volumes and structural similarities were derived from the same 


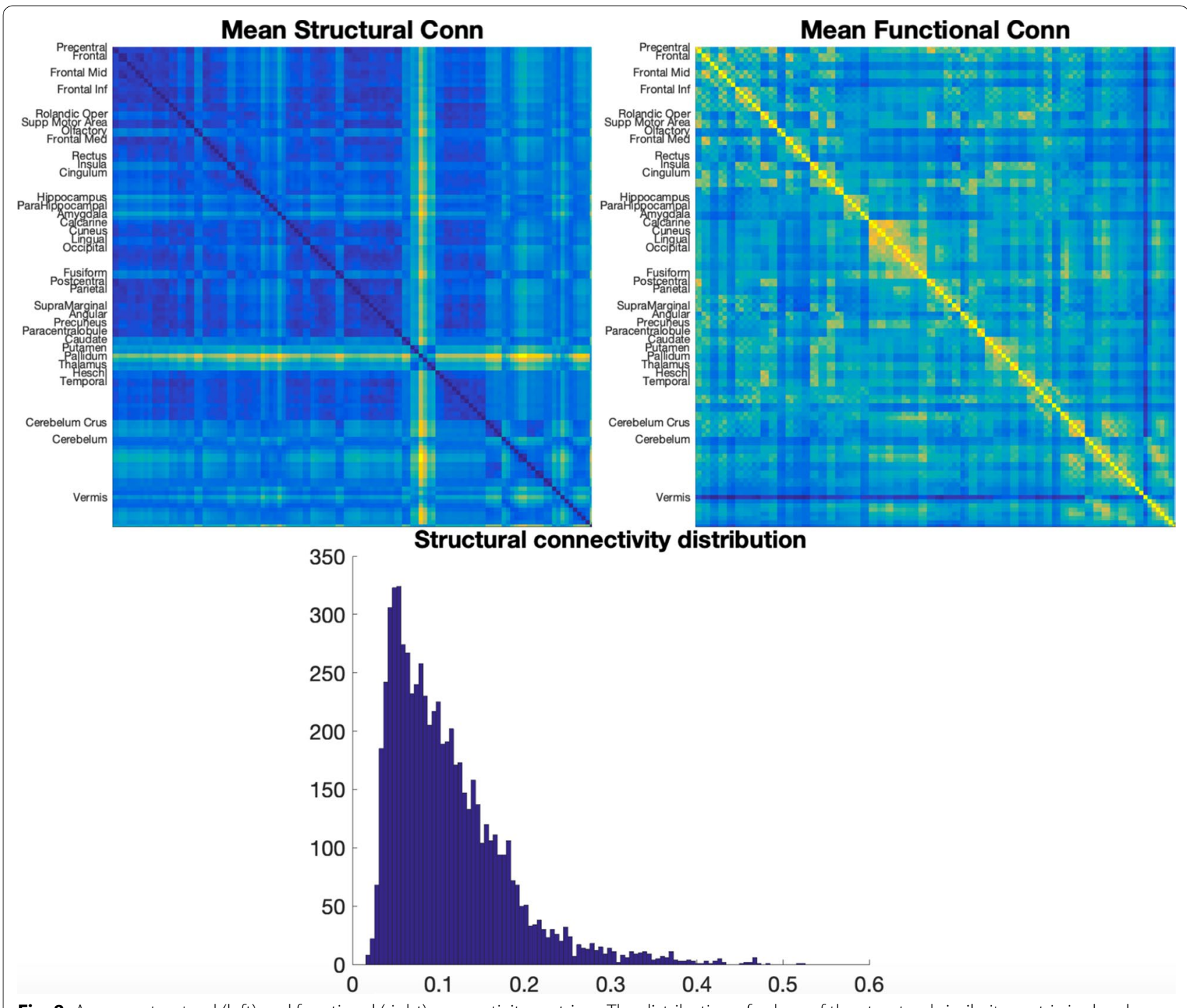

Fig. 8 Average structural (left) and functional (right) connectivity matrices. The distribution of values of the structural similarity metric is also shown

imaging data. This brings up three key points. First, the method of encoding data is important because it presents different types of information to the machine learning model. Structural differences in autism (and likely other phenotypic differences) may vary in different ways that are only apparent under specific methods of encoding, and thus, the model may have focused on different areas, depending on which method of encoding was performed. This is important for both interpreting the results in the context of a specific machine learning task and understanding the underlying physiological implications. Second, the emphases presented by Grad-CAM were relative; that is, in analysing the distribution of Grad-CAM values, we saw that the model took all areas into account (Fig. 7), although with highest focus on the few areas that seemed to hold more influence in the final classification task. This does not, however, mean that other areas were ignored entirely. Third, because of the higher dimensionality of structural similarities over grey matter values, it may be the case that the machine learning model assumed information about grey matter volumes from a small number of edges, while information about differences in morphology of other areas (e.g. the left and right Heschl's gyrus), which were not present in the univariate feature extractions, required emphasis by a greater number of edges; this may be crucial to understanding differences in autism generally, or it may have simply helped the model increase AUROC by a margin of 0.0891 between the univariate and connectivity classification tasks. Stated informally, differences in morphology detected by the structural similarity matrices were more subtle, and so they required the emphasis of a larger number of edges. 
The results in Fig. 3 show that autism classification, even when structure and function are considered, generalizes poorly across large age groups. This supports the findings in recent longitudinal studies of autism [70-72], which found high inter-individual variability in brain volume growth trajectories in autism. This suggests that autism is highly variable in its development and that information about one age group with autism would not necessarily inform predictions of another age group. To properly generalize these findings in the context of this study, more data from older age groups would be needed.

The differences between ensemble AUROCs are small in some cases, but these represent the convergent AUROC of 300 individual models rather than a single trained model, which would be more subject to classification error. Evidence for this is shown in Fig. 3, which shows a predictable convergence of test set accuracy as more models are added to an ensemble. This indicates that the observed AUROCs are reproducible in the context of this study, though further analyses are necessary to form strong conclusions about the neurobiology of autism.

Because of this, we can be sure that the AUROCs represented for each set of features are reproducible, and while the advantages of integrating multiple sets of features does lead to increased performance, this increase is only slight. This may indicate an upper limit on possible test set accuracy given the features represented in the data; in other words, the presented data may simply not have patterns present that can generally indicate differences between autism and controls, and so integrating more features into a deep learning model has diminishing returns.

It is notable that none of the classification accuracies presented in this paper approached the success required for a clinical diagnosis, which would need to consistently exceed $95 \%$ accuracy on a substantially large dataset. A likely reason for the comparatively low accuracy in this study specifically is the large dataset size, which, in the context of whole-brain MRI classification, has previously been associated with a drop in accuracy [50, 73], as well as the possible lack of neurobiological signatures that would be able to sufficiently distinguish autist subjects from typical controls. Nonetheless, deep learning models are useful in these contexts both as statistical models in and of themselves to study autism, and as building blocks to approach clinical-quality accuracy in the future.

Finally, we combined our structural similarity metric with functional connectivity raising the final AUROC. This shows that our method does not have to be considered as a replacement for any previous methods, but may be used in combination with them in order to make single-participant classifications more effective.

\section{Limitations}

This study has several limitations. While we used a large sample size, most data were drawn from a relatively narrow age group, and so generalizations of the findings to adult autism may be inappropriate. Additionally, with the extreme heterogeneity of autism and the different sites and studies the data was drawn from, as well as varying reliability of autism labels depending on diagnostic criteria in different sites, a binary machine learning task of the type described here may be ill-formed. Nonetheless, given lack of richer information within the data, options for design of the machine learning problem are limited. Finally, insights into areas of the brain deemed salient by the deep learning visualization methods may be specific to the methods used to derive the brain connectome, or to a specific subgroup in the data, and further studies are required to assess the generalizability of these findings.

\section{Conclusion}

The present study offers a means of encoding T1-weighted MRI for use in network-based machine learning models, and with a machine learning classification task we have demonstrated an increase in accuracy in classifying autistic individuals when compared with both functional connectivities and classification of univariate grey matter volumes. Furthermore, we presented methods of identified areas emphasized by the machine learning model, demonstrating the importance of data encoding and highlighting complications with interpreting results when the feature extractions have no specific physiological interpretation. While this trade-off, interpretability for higher accuracy, will likely continue to be an issue in machine learning with scientific data, the effects of data encoding on accuracy point towards feature extraction methods as a future direction of investigation.

\section{Abbreviations \\ ABCD: Adolescent brain cognitive development; ABIDE: Autism brain imag- ing data exchange; AFNl: Analysis of functional neuroimages; ASD: Autism spectrum disorder; BOLD: Blood oxygen level-dependent; CAM: Class activa- tion map; CNN: Convolutional neural network; CSF: Cerebrospinal fluid; DWl: Diffusion-weighted imaging; fMRI: Functional magnetic resonance imag- ing; Grad-CAM: Gradient class activation map; MNI: Montreal neurological institute; MRI: Magnetic resonance imaging; NDAR: National database for ASD research; ReLU: Rectified linear unit; ROI: Region of interest; VBM: Voxel-based morphometry.}

\section{Acknowledgements}

NDAR Acknowledgement: Data and/or research tools used in the preparation of this manuscript were obtained from the $\mathrm{NIH}$-supported National Database for ASD Research (NDAR). NDAR is a collaborative informatics system created by the National Institutes of Health to provide a national resource to support and accelerate research in ASD. Dataset identifier(s): [NIMH Data Archive Collection ID(s) or NIMH Data Archive Digital Object Identifier (DOI)]. This manuscript reflects the views of the authors and may not reflect the opinions or views of the $\mathrm{NIH}$ or of the Submitters submitting original data to NDAR. ABCD 
Acknowledgement: Data used in the preparation of this article were obtained from the Adolescent Brain Cognitive Development (ABCD) Study (https:// abcdstudy.org), held in the NIMH Data Archive (NDA). This is a multisite, longitudinal study designed to recruit more than 10,000 children age 9-10 and follow them over 10 years into early adulthood. The ABCD Study is supported by the National Institutes of Health and additional federal partners under award numbers U01DA041022, U01DA041028, U01DA041048, U01DA041089, U01DA041106, U01DA041117, U01DA041120, U01DA041134, U01DA041148, U01DA041156, U01DA041174, U24DA041123, and U24DA041147. A full list of supporters is available at https://abcdstudy.org/federal-partners.html. A listing of participating sites and a complete listing of the study investigators can be found at https://abcdstudy.org/Consortium_Members.pdf. ABCD consortium investigators designed and implemented the study and/or provided data but did not necessarily participate in analysis or writing of this report. This manuscript reflects the views of the authors and may not reflect the opinions or views of the NIH or ABCD consortium investigators. UK Biobank Acknowledgement: This research has been conducted using the UK Biobank Resource [project ID 20904]. Other database Acknowledgements: We would also like to thank ABIDE I, ABIDE II, and Open fMRI for the use of their data.

\section{Authors' contributions}

M. Leming performed experiments, analysed data, and drafted the manuscript; M. Leming and J. Suckling conceived project; S. Baron-Cohen provided expertise on autism and contributed to interpretation of the findings. All authors read and approved final manuscript.

\section{Funding}

This research was co-funded by the NIHR Cambridge Biomedical Research Centre and Marmaduke Sheild. Matthew Leming was supported by a Gates Cambridge Scholarship from the University of Cambridge. This research was co-funded by the NIHR Cambridge Biomedical Research Centre and a Marmaduke Sheild grant to Richard A.I. Bethlehem and Varun Warrier. The views expressed are those of the author(s) and not necessarily those of the NHS, the NIHR or the Department of Health and Social Care.

\section{Availability of data and materials}

All data analysed in this article is public. Code for analysis is available on Github.

\section{Declarations}

\section{Ethics approval and consent to participate}

Not applicable.

\section{Consent for publication}

Not applicable.

\section{Competing interests}

The authors declare that they have no competing interests.

\section{Author details}

${ }^{1}$ Department of Psychiatry, University of Cambridge, Robinson Way, Cambridge, Cambridgeshire CB2 0SZ, UK. ${ }^{2}$ Present Address: Center for Systems Biology, Massachusetts General Hospital, 149 13th Street, Boston, MA 02129, USA.

Received: 3 November 2020 Accepted: 16 April 2021

Published online: 10 May 2021

\section{References}

1. Whitwell JL. Voxel-based morphometry: an automated technique for assessing structural changes in the brain. J Neurosci. 2009;29:9661-4. https://doi.org/10.1523/JNEUROSCI.2160-09.2009.

2. Wright IC, MCGuire PK, Poline JB, Travere JM, Murray RM, Frith CD, Frackowiak RS, Friston KJ. A voxel-based method for the statistical analysis of gray and white matter density applied to schizophrenia. Neuroimage. 1995;2:244-52. https://doi.org/10.1006/nimg.1995.1032.
3. Ashburner J, Friston KJ. Voxel-based morphometry: the methods. Neuroimage. 2000;11:805-21. https://doi.org/10.1006/nimg.2000.0582.

4. Mechelli A, Friston KJ, Frackowiak RS, Price CJ. Structural covariance in the human cortex. J Neurosci. 2005;25:8303-10. https://doi.org/10.1523/ JNEUROSCI.0357-05.2005.

5. Romero-Garcia R, Whitaker KJ, Váša F, Seidlitz J, Shinn M, Fonagy P, Dolan RJ, Jones PB, Goodyer IM.the NSPN Consortium, Bullmore ET, Vértes PE. Structural covariance networks are coupled to expression of genes enriched in supragranular layers of the human cortex. Neurolmage $\mathbf{1 7 1}$ (2018). https://doi.org/10.1016/j.neuroimage.2017.12.060

6. Alexander-Bloch AF, Vértes PE, Stidd R, Lalonde F, Clasen L, Rapoport J, Giedd J, Bullmore ET, Gogtay N. The anatomical distance of functional connections predicts brain network topology in health and schizophrenia. Cereb Cortex. 2013;23:127-38. https://doi.org/10.1093/cercor/ bhr388.

7. Redcay E, Courchesne E. Biol Psychiatry. 2005;58:1-9. https://doi.org/10. 1016/j.biopsych.2005.03.026.

8. Stanfield AC, McIntosh AM, Spencer MD, Philip R, Gaur S, Lawrie SM. Towards a neuroanatomy of autism: a systematic review and metaanalysis of structural magnetic resonance imaging studies. Eur Psychiatry. 2008;23:289-99. https://doi.org/10.1016/j.eurpsy.2007.05.006.

9. Nickl-Jockschat T, Habel U, Michel TM, Manning J, Laird AR, Fox PT, Schneider F, Eickhoff SB. Brain structure anomalies in autism spectrum disordera meta-analysis of vbm studies using anatomic likelihood estimation. Hum Brain Mapp. 2012;33:1470-89. https://doi.org/10.1002/hbm.21299.

10. Haar S, Berman S, Behrmann M, Dinstein I. Anatomical abnormalities in autism? Cereb Cortex. 2016;26:1440-52. https://doi.org/10.1093/cercor/ bhu242.

11. Chen R, Jiao Y, Herskovits E. Structural MRI in autism spectrum disorder. Pediatr Res. 2011;69:63-8. https://doi.org/10.1203/PDR.0b013e3182 $12 \mathrm{c} 2 \mathrm{~b} 3$.

12. Sparks BF, Friedman SD, Shaw DW, Aylward EH, Echelard D, Artru AA, Maravilla KR, Giedd JN, Munson J, Dawson G, Dager SR. Brain structural abnormalities in young children with autism spectrum disorder. Neurology. 2002;59:184-92. https://doi.org/10.1212/wnl.59.2.184.

13. Schumann CM, Hamstra J, Goodlin-Jones BL, Lotspeich LJ, Kwon H, Buonocore MH, Lammers CR, Reiss AL, Amaral DG. The amygdala is enlarged in children but not adolescents with autism; the hippocampus is enlarged at all ages. J Neurosci. 2004;24:6392-401. https://doi.org/10. 1523/JNEUROSCI.1297-04.2004.

14. Cardon GJ, Hepburn S, Rojas DC. Structural covariance of sensory networks, the cerebellum, and amygdala in autism spectrum disorder. Front Neurol. 2017. https://doi.org/10.3389/fneur.2017.00615.

15. McAlonan GM, Cheung V, Cheung C, Suckling J, Lam GY, Tai KS, Yip L, Murphy DG, Chua SE. Mapping the brain in autism: a voxel-based MR study of volumetric differences and intercorrelations in autism. Brain. 2005;128(Pt 2):268-76. https://doi.org/10.1093/brain/awh332.

16. Sharda M, Foster NEV, Tryfon A, Doyle-Thomas KAR, Ouimet T, Anagnostou E, Evans AC, Zwaigenbaum L, Lerch JP, Lewis JD, Hyde KL, Group, NAI. Language ability predicts cortical structure and covariance in boys with autism spectrum disorder. Cereb Cortex 27, 1849-1862 (2017). https:// doi.org/10.1093/cercor/bhw024.

17. Prigge MD, Bigler ED, Fletcher PT, Zielinski BA, Ravichandran C, Anderson J, Froehlich A, Abildskov T, Papadopolous E, Maasberg K, Nielsen JA, Alexander $\mathrm{AL}$, Lange $\mathrm{N}$, Lainhart J. Longitudinal heschls gyrus growth during childhood and adolescence in typical development and autism. Autism Res. 2013;6:78-90. https://doi.org/10.1002/aur.1265.

18. Hurlburt RT, Alderson-Day B, Kühn S, Fernyhough C. Exploring the ecological validity of thinking on demand: neural correlates of elicited vs. spontaneously occurring inner speech. PLoS ONE. 2016;11:0147932. https://doi.org/10.1371/journal.pone.0147932.

19. Balardin, J.B., Comfort, W.E., Daly, E., Murphy, C., Andrews, D., Murphy, D.G., Ecker, C., Consortium, M.A., Sato, J.R. Decreased centrality of cortical volume covariance networks in autism spectrum disorders. J Psychiatr Res. 2015;69:142-9. https://doi.org/10.1016/j.jpsychires.2015.08.003.

20. Simas T, Chattopadhyay S, Hagan C, Kundu P, Patel A, Holt R, Floris D, Graham J, Ooi C, Tait R, Spencer M, Baron-Cohen S, Sahakian B, Bullmore E, Goodyer I, Suckling J. Semi-metric topology of the human connectome: sensitivity and specificity to autism and major depressive disorder. PLoS ONE. 2015. https://doi.org/10.1371/journal.pone.0136388. 
21. Müller E, Schuler A, Yates GB. Social challenges and supports from the perspective of individuals with asperger syndrome and other autism spectrum disabilities. Autism. 2008;12:173-90. https://doi.org/10.1177/ 1362361307086664

22. Just MA, Cherkassky VL, Keller TA, Minshew NJ. Cortical activation and synchronization during sentence comprehension in high-functioning autism: evidence of underconnectivity. Brain. 2004;127:1811-21. https:// doi.org/10.1093/brain/awh199.

23. Cherkassky VL, Kana RK, Keller TA, Just MA. Functional connectivity in a baseline resting-state network in autism. Neuroreport. 2006;17:1687-90. https://doi.org/10.1097/01.wnr.0000239956.45448.4c.

24. Kennedy DP, Courchesne E. The intrinsic functional organization of the brain is altered in autism. Neurolmage. 2008;39:1877-85. https://doi.org/ 10.1016/j.neuroimage.2007.10.052

25. Assaf M, Jagannathan K, Calhoun VD, Miller L, Stevens MC, Sahl R, O'Boyle JG, Schultz RT, Pearlson GD. Abnormal functional connectivity of default mode sub-networks in autism spectrum disorder patients. Neurolmage. 2010;53:247-56. https://doi.org/10.1016/j.neuroimage.2010.05.067.

26. Jones TB, Bandettini PA, Kenworthy L, Case LK, Milleville SC, Martin A, Birn RM. Sources of group differences in functional connectivity: an investigation applied to autism spectrum disorder. Neurolmage. 2010;49:401-14. https://doi.org/10.1016/j.neuroimage.2009.07.051.

27. Weng SJ, Wiggins JL, Peltier SJ, Carrasco M, Risi S, Lord C, Monk CS. Alterations of resting state functional connectivity in the default network in adolescents with autism spectrum disorders. Brain Res. 2010;1313:20214. https://doi.org/10.1016/j.brainres.2009.11.057

28. Cerliani L, Mennes M, Thomas RM, Martino A, Thioux M, Keysers C. Increased functional connectivity between subcortical and cortical resting-state networks in autism spectrum disorder. JAMA Psychiatry. 2015;72:767-77. https://doi.org/10.1001/jamapsychiatry.2015.0101.

29. Chien HY, Lin HY, Lai MC, Gau SS, Tseng WY. Hyperconnectivity of the right posterior temporo-parietal junction predicts social difficulties in boys with autism spectrum disorder. Autism Res. 2015;8:427-41. https:// doi.org/10.1002/aur.1457.

30. Delmonte S, O'Gallagher L, Hanlon E, McGrath J, Balsters JH. Functional and structural connectivity of frontostriatal circuitry in autism spectrum disorder. Front Hum Neurosci. 2013;7:430. https://doi.org/10.3389/fnhum. 2013.00430.

31. Di Martino A, Kelly C, Grzadzinski R, Zuo XN, Mennes M, Mairena MA, Lord C, Castellanos FX, Milham MP. Aberrant striatal functional connectivity in children with autism. Biol Psychiatry. 2011;69:847-56. https://doi.org/10. 1016/j.biopsych.2010.10.029.

32. Nebel MB, Eloyan A, Barber AD, Mostofsky SH. Precentral gyrus functional connectivity signatures of autism. Front Syst Neurosci. 2014;8:80. https:// doi.org/10.3389/fnsys.2014.00080.

33. Nebel MB, Joel SE, Muschelli J, Barber AD, Caffo BS, Pekar JJ, Mostofsky SH. Disruption of functional organization within the primary motor cortex in children with autism. Hum Brain Mapp. 2014;35:567-80. https://doi.org/ 10.1002/hbm.22188.

34. Hull JV, Dokovna LB, Jacokes ZJ, Torgerson CM, Irimia A, van Horn JD. Resting-state functional connectivity in autism spectrum disorders: a review. Front Psychiatry. 2017. https://doi.org/10.3389/fpsyt.2016.0020.

35. Behrens TEJ, Berg HJ, Jbabdi S, Rushworth MFS, Woolrich M. Probabilistic diffusion tractography with multiple fibre orientations: What can we gain? Neurolmage. 2007;34:144-55. https://doi.org/10.1016/j.neuro image.2006.09.018.

36. Kriston KJ. Functional and effective connectivity: a review. Brain Connectivity. 2011;1:13-36. https://doi.org/10.1089/brain.2011.0008.

37. Meszlényi R, Buza K, Vidnyánszky Z. Resting state FMRI functional connectivity-based classification using a convolutional neural network architecture. Front Neuroinform. 2017;11:61. https://doi.org/10.3389/fninf. 2017.00061.

38. Kazeminejad A, Sotero RC. Topological properties of resting-state fmri functional networks improve machine learning-based autism classification. Front Neurosci. 2019. https://doi.org/10.3389/fnins.2018.01018.

39. Al-Zubaidi A, Mertins A, Heldmann M, Jauch-Chara K, Münte TF. Machine learning based classification of resting-state FMRI features exemplified by metabolic state (hunger/satiety). Front Hum Neurosci. 2019. https://doi. org/10.3389/fnhum.2019.00164

40. Dodonova Y, Korolev S, Tkachev A, Petrov D. Classification of structural brain networks based on information divergence of graph spectra. In:
2016 IEEE 26th international workshop on machine learning for signal processing (MLSP) (2016). https://doi.org/10.1109/MLSP.2016.7738852.

41. Kawahara J, Brown CJ, Miller SP, Booth BG, Chau V, Grunau RE, Zwicker JG, Hamarneh G. Brainnetcnn: convolutional neural networks for brain networks; towards predicting neurodevelopment. Neurolmage. 2017;146:1038-49. https://doi.org/10.1016/j.neuroimage.2016.09.046.

42. Frau-Pascual A, Fogarty M, Fischl B, Yendiki A, Aganj I. Quantification of structural brain connectivity via a conductance model. Neurolmage. 2019;189:485-96. https://doi.org/10.1016/j.neuroimage.2019.01.033.

43. Patel AX, Bullmore ET. A wavelet-based estimator of the degrees of freedom in denoised fmri time series for probabilistic testing of functional connectivity and brain graphs. Neurolmage. 2016;142:14-26. https://doi. org/10.1016/j.neuroimage.2015.04.052.

44. Seidlitz J, Váša F, Shinn M, Romero-Garcia R, Whitaker KJ, Vértes PE, Wagstyl K, Kirkpatrick Reardon P, Clasen L, Liu S, Messinger A, Leopold DA, Fonagy P, Dolan RJ, Jones PB, Goodyer IM, Consortium N, Raznahan A, Bullmore ET. Morphometric similarity networks detect microscale cortical organization and predict inter-individual cognitive variation. Neuron. 2018;97:231-2477. https://doi.org/10.1016/j.neuron.2017.11.039.

45. Paquola C, Vos De Wael R, Wagstyl K, Bethlehem RAI, Hong S-J, Seidlitz J, Bullmore ET, Evans AC, Misic B, Margulies DS, Smallwood J, Bernhardt BC. Microstructural and functional gradients are increasingly dissociated in transmodal cortices. PLoS Biol 17, 3000284 (2019). https://doi.org/10. 1371/journal.pbio.3000284.

46. Kong XZ, Wang X, Huang L, Pu Y, Yang Z, Dang X, Zhen Z, Liu J. Measuring individual morphological relationship of cortical regions. J Neurosci Methods. 2014;237:103-7. https://doi.org/10.1016/j.jneumeth.2014.09. 003.

47. Kong XZ, Liu Z, Huang L, Wang X, Yang Z, Zhou G, Zhen Z, Liu J. Mapping individual brain networks using statistical similarity in regional morphology from MRI. PLoS ONE. 2015;10:0141840. https://doi.org/10.1371/journ al.pone.0141840.

48. Preston DC. Magnetic resonance imaging (MRI) of the brain and spine: basics. Case Western Reserve University School of Medicine (2006). https://casemed.case.edu/clerkships/neurology/Web\%20Neurorad/MRI\% 20Basics.htm Accessed 30-03-2020.

49. Plitt M, Barnes KA, Martin A. Functional connectivity classification of autism identifies highly predictive brain features but falls short of biomarker standards. Neurolmage Clin. 2015;7:359-66. https://doi.org/10. 1016/j.nicl.2014.12.013.

50. Katuwal GJ, Cahill ND, Baum SA, Michael AM. The predictive power of structural mri in autism diagnosis. Conf Proc IEEE Eng Med Biol Soc. 2015;4270-4273. https://doi.org/10.1109/EMBC.2015.7319338.

51. Heinsfeld AS, Franco AR, Craddock RC, Buchweitz A, Meneguzzia F. Identification of autism spectrum disorder using deep learning and the abide dataset. Neurolmage: Clinical 17, 16-23 (2018). https://doi.org/10.1016/j. nicl.2017.08.017.

52. Khosla KM, An J, Kuceyeski A, Sabuncu MR. 3d convolutional neural networks for classification of functional connectomes. MICCAI 2018 (2018). https://doi.org/10.1007/978-3-030-00889-5_16.

53. Hazlett HC, Gu H, Munsell BC, Kim SH, Styner M, Wolff JJ, Elison JT, Swanson MR, Zhu H, Botteron KN, Collins DL, Constantino JN, Dager SR, Estes AM, Evans AC, Fonov VS, Gerig G, Kostopoulos P, McKinstry RC, Pandey J, Paterson S, Pruett JR, Schultz RT, Shaw DW, Zwaigenbaum L, Piven J. Early brain development in infants at high risk for autism spectrum disorder. Nature. 2017;542:348-51. https://doi.org/10.1038/nature21369.

54. Emerson RW, Adams C, Nishino T, Hazlett HC, Wolff JJ, Zwaigenbaum L, Constantino JN, Shen MD, Swanson MR, Elison JT, Kandala S, Estes AM, Botteron KN, Collins L, Dager SR, Evans AC, Gerig G, Gu H, McKinstry RC, Paterson S, Schultz RT, Styner M, Schlaggar BL, Pruett JR, Piven J. Functional neuroimaging of high-risk 6-month-old infants predicts a diagnosis of autism at 24 months of age. Sci Transl Med. 2017;9:2882. https://doi. org/10.1126/scitranslmed.aag2882.

55. Anderson JS, Druzgal TJ, Froehlich A, DuBray MB, Lange N, Alexander AL, Abildskov T, Nielsen JA, Cariello AN, Cooperrider JR, Bigler ED, Lainhart JE. Decreased interhemispheric functional connectivity in autism. Cereb Cortex. 2011;21:1134-46. https://doi.org/10.1093/cercor/bhq190.

56. Barttfeld P, Wicker B, Cukier S, Navarta S, Lew S, Leiguarda R, Sigman M. State-dependent changes of connectivity patterns and functional brain network topology in autism spectrum disorder. Neuropsychologia. 
2012;50:3653-62. https://doi.org/10.1016/j.neuropsychologia.2012.09. 047.

57. Nielsen JA, Zielinski BA, Fletcher PT, Alexander AL, Lange N, Bigler ED, Lainhart JSJE. anderson: multisite functional connectivity MRI classification of autism: Abide results. Front Hum Neurosci. 2013;7:599. https://doi. org/10.3389/fnhum.2013.00599.

58. Jung M, Kosaka H, Saito DN, Ishitobi M, Morita T, Inohara K, Asano M, Arai S, Munesue T, Tomoda A, Wada Y, Sadato N, Okazawa H, lidaka T. Default mode network in young male adults with autism spectrum disorder: relationship with autism spectrum traits. Mol Autism. 2014;5:35. https:// doi.org/10.1186/2040-2392-5-35.

59. lidaka T. Resting state functional magnetic resonance imaging and neural network classified autism and control. Cortex. 2015;63:55-67. https://doi. org/10.1016/j.cortex.2014.08.011.

60. Tejwani R, Liska A, You H, Reinen J, Das P. Autism classification using brain functional connectivity dynamics and machine learning. ArXiv (2017)

61. Eill A, Jahedi A, Gao Y, Kohli JS, Fong CH, Solders S, Carper RA, Valafar F, Bailey BA, Müller R. Functional connectivities are more informative than anatomical variables in diagnostic classification of autism. Brain Connectivity. 2019. https://doi.org/10.1089/brain.2019.0689.

62. Leming M, Suckling J. Stochastic encoding of graphs in deep learning allows for complex analysis of gender classification in resting-state and task functional brain networks from the UK biobank. arXiv (2020)

63. Patel AX, Kundu P, Rubinov M, Jones PS, Vertes PE, Ersche KD, Suckling J, Bullmore ET. A wavelet method for modeling and despiking motion artifacts from resting-state FMRI time series. Neurolmage. 2014;95:287-304.

64. Ramdas A, Garcia N, Cuturi M. On wasserstein two sample testing and related families of nonparametric tests. Entropy. 2017;19:47. https://doi. org/10.3390/e19020047.

65. Rubner Y, Tomasi C, Guibas $\sqcup$. The earth movers distance as a metric for image retrieval. Int J Comput Vis. 2000:40:99-121. https://doi.org/10. 1023/A:1026543900054.
66. Selvaraju RR, Cogswell M, Das A, Vedantam R, Parikh D, Batra D. Grad-cam: visual explanations from deep networks via gradient-based localization. In: 2017 IEEE international conference on computer vision (ICCV) (2017). https://doi.org/10.1109/ICCV.2017.74.

67. Rubinov M, Sporns O. Complex network measures of brain connectivity: uses and interpretations. Neurolmage. 2010;52:1059-69.

68. Tijms BM, Seriès P, Willshaw DJ, Lawrie SM. Similarity-based extraction of individual networks from gray matter MRI scans. Cereb Cortex. 2012;22:1530-41. https://doi.org/10.1093/cercor/bhr221.

69. Suckling J, Simas T, Chattopadhyay S, Tait R, Su L, Williams G, Rowe JB, O'Brien JT. A winding road Alzheimers disease increases circuitous functional connectivity pathways. Front Comput Neurosci. 2015. https://doi. org/10.3389/fncom.2015.00140.

70. Ha S, Sohn IJ, Kim N, Sim HJ, Cheon KA. Characteristics of brains in autism spectrum disorder: structure, function and connectivity across the lifespan. Exp Neurobiol. 2015;24:273-84. https://doi.org/10.5607/en.2015. 24.4.273.

71. Lange N, Travers BG, Bigler ED, Prigge MB, Froehlich AL, Nielsen JA, et al. Longitudinal volumetric brain changes in autism spectrum disorder ages 6-35 years. Autism Res. 2015;8:82-93. https://doi.org/10.1002/aur.1427.

72. Wolff JJ, Jacob S, Elison JT. The journey to autism: insights from neuroimaging studies of infants and toddlers. Dev Psychopathol. 2018;30:479-95. https://doi.org/10.1017/S0954579417000980.

73. Arbabshirani MR, Plis S, Sui J, Calhoun VD. Single subject prediction of brain disorders in neuroimaging: promises and pitfalls. Neurolmage. 2017;145(Pt B):137-65. https://doi.org/10.1016/j.neuroimage.2016.02.079.

\section{Publisher's Note}

Springer Nature remains neutral with regard to jurisdictional claims in published maps and institutional affiliations.
Ready to submit your research? Choose BMC and benefit from:

- fast, convenient online submission

- thorough peer review by experienced researchers in your field

- rapid publication on acceptance

- support for research data, including large and complex data types

- gold Open Access which fosters wider collaboration and increased citations

- maximum visibility for your research: over $100 \mathrm{M}$ website views per year

At BMC, research is always in progress.

Learn more biomedcentral.com/submissions 